\title{
Chronic renal failure as initial presentation of pelvic hemangioendothelioma.
}

\author{
Insuficiência renal crônica como manifestação inicial de hemangioendotelioma \\ pélvico.
}

João Gabriel Léda Braga ${ }^{1 \oplus}$, Fernanda Monteiro Orellana ${ }^{2 \oplus}$, Alan Ziroldo ${ }^{3 \oplus}$, Leonardo Sementilli4 ${ }^{4}$, Luis Gustavo Morato De Toledo ${ }^{2 \oplus}$, Roni de Carvalho Fernandes ${ }^{2}$.

\begin{abstract}
Hemangioendothelioma is a rare vascular tumor whose etiology has not yet been defined. Currently, it is known to affect different sites, such as the abdominal-pelvic cavity. It is also known to present intermediate characteristics to hemangioma and angiosarcoma. In recent studies, $50-76 \%$ of patients are asymptomatic. Surgical treatment is indicated in most cases. The authors present a case of hemangioendothelioma with pelvic involvement and initial presentation as acute renal failure due to extrinsic ureteral compression, treated surgically, with histopathological diagnosis of hemangioendothelioma and recurrence after one year of the initial treatment presenting a histologically different form. This case illustrates a rare cause of lower urinary tract symptoms (LUTS), obstructive uropathy and acute renal failure and the appearance of two different tumors within a year, in line with the genetic/immunological factor in its genesis.
\end{abstract}

Keywords: Hemangioendothelioma; Sarcoma; Myxoid liposarcoma; Chronic renal failure..

1. Santa Casa de Misericórdia de São Paulo, General Surgery, São Paulo, São Paulo, Brazil.

2. Santa Casa de Misericórdia de São Paulo, Urology - São Paulo, São Paulo, Brazil.

3. Santa Casa de São Paulo School of Medical Sciences, Medicine, São Paulo, São Paulo, Brazil.

4. Santa Casa de Misericórdia de São Paulo, Pathology - São Paulo - São Paulo - Brazil.

Financial support: none to declare.

Conflicts of interest: The authors declare no conflict of interest relevant to this manuscript.

Correspondence author: João Gabriel Léda Braga, Santa Casa De Misericórdia De São Paulo, General Surgery - São Paulo - São Paulo - Brazil

E-mail: joaogabriellbraga@gmail.com 


\section{RESUMO}

O hemangioendotelioma é um tumor vascular raro cuja etiologia ainda não foi definida. Atualmente, sabe-se que afeta diferentes locais, como a cavidade abdominal e pélvica. Sabe-se também que possui características intermediárias do hemangioma e do angiossarcoma. Em estudos recentes, $50-76 \%$ dos pacientes são assintomáticos. O tratamento cirúrgico é indicado na maioria dos casos. Os autores apresentam um caso de hemangioendotelioma com envolvimento pélvico e apresentação inicial como insuficiência renal aguda por compressão extrínseca ureteral, tratado cirurgicamente, diagnóstico histopatológico de hemangioendotelioma e recorrência após um ano do tratamento inicial, apresentando uma forma histologicamente diferente. Este caso ilustra uma causa rara de LUTS, uropatia obstrutiva e insuficiência renal aguda e o aparecimento de dois tumores diferentes dentro de um ano, em consonância com o fator genético/imunológico em sua gênese.

Descritores: Hemangioendotelioma; Sarcoma; Lipossarcoma mixóide; Insuficiência renal crônica.

\section{INTRODUCTION}

Hemangioendothelioma is a vascular tumor with an epithelioid and histiocytoid aspect that originates from vascular endothelial or pre-endothelial cells. It is a rare vascular tumor, representing less than $1 \%$ of all vascular tumors, being first described by Dail and Liebow, in 1975x, as a highly aggressive bronchoalveolar cell carcinoma, currently known that it can affect different sites, such as the abdominal-pelvic cavity and that it has intermediate characteristics to hemangioma and angiosarcoma. Estimates that $50-76 \%$ of patients are asymptomatic at the time of diagnosis. ${ }^{[1]}$

Etiology is not yet defined. At the molecular level, characterized by recurrent translocations involving the chromosomal regions 1 p36.3 and 3q25; the resulting translocation between the WWTR1 (transcriptional regulator 1 of the WW domain; 3q25) and CAMTA1 (transcriptional activator of the binding to calmodulin $1 ; 1 \mathrm{p36}$ ) results in the formation of a WWTR1/CAMTA1 fusion gene that defines the disease. ${ }^{[2]}$

Another hypothesis for the pathogenesis of this tumor is related to chronic infection by Bartonella spp. Its capability to invade and induce long-lasting intra-erythrocytic and intra-endothelial infections, along with the ability of at least three Bartonella spp. (B. henselae, B. quintana, and B. bacilliformis), causes vasoproliferation mediated by the vascular endothelial growth factor, suggest that these bacteria may contribute to the development of these tumors. ${ }^{[1]}$

It is often accidentally diagnosed, and more than 50$76 \%$ of patients are asymptomatic, usually detected in imaging exams to diagnose other pathologies or even in routine exams. A small proportion of patients have only minor and non-specific symptoms at the time of diagnosis. The lesion is usually multiple and variable size. ${ }^{[1]}$

Hemangioendothelioma has no defined standard of treatment, and few therapeutic options are available. ${ }^{[1]}$ Surgical treatment is indicated for nonmetastatic disease, aiming for the complete resection of the tumor. For metastatic diseases, chemotherapy is the most used treatment, yet there is little evidence of success. ${ }^{[3]}$ Our case highlights a rare clinical presentation, etiopathogenesis, diagnosis, and treatment of pelvic hemangioendothelioma.

\section{CASE REPORT}

A 70-year-old Caucasian man presented to the urology emergency department of Santa Casa de Sao Paulo due to post-renal failure and oligoanuria. He had been on dialysis by Shiley catheter for 15 days according to metabolic demand. Among the reported symptoms, he presented a dragged profile of significant weight loss, lack of appetite, post-voiding drip, straining urination, urgency incontinence, and nocturia (5x/night). A personal history of tobacco use: 10 pack-years. On physical examination, we observed a malnourished patient using an indwelling bladder catheter. The abdomen was hollowed out, flabby, painless, and without palpable masses. Rectal examination with fibroelastic prostate, approximately $30 \mathrm{~g}$ and a palpable mass to the left of the prostate, of cystic consistency without clear upper limits. Laboratory tests showed $7.04 \mathrm{ng} / \mathrm{ml}$ prostatic specific antigen (PSA) (24\% free PSA ratio), 212mg/dl urea, 9.4mg/dl serum creatinine, $8.1 \mathrm{~g} / \mathrm{dl}$ hemoglobin.

Ultrasound (USG) was performed and showed bilateral hydronephrosis. The patient underwent abdominal computed tomography (CT) with no contrast, which showed an $8 \times 7.5 \times 7.4 \mathrm{~cm}$ lateral pelvic mass next to the prostate. It was a cystic component, well-defined, rounded, with internal septations and calcifications, promoting an expansive effect and contralateral displacement of the prostate and left seminal vesicle, with no signs of local invasion or lymph node enlargement (Figure 1), displacing the bladder laterally, promoting mass effect, and bilateral ureteral compression.

Nephrologists kept the dialysis indication due to the increase in nitrogen slags and anuria. Cystoscopy 


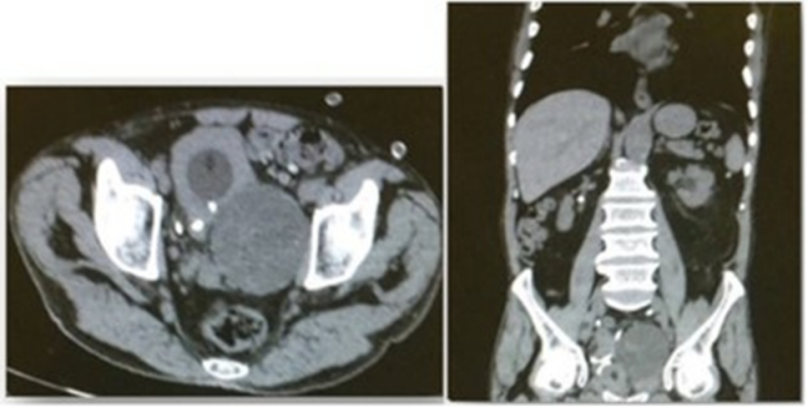

Figure 1. Axial and coronal section, respectively, of CT scan with no contrast of the pelvis showing an $8 \times 7.5 \times 7.4 \mathrm{~cm}$ lateral pelvic mass, next to the prostate, cystic component, well-defined, rounded, with internal septations and calcifications.

showed a very thickened and trabeculated bladder, with a bulge on its floor and left side. The catheter inserted was a bilateral double-J. The patient maintained an increase in creatinine and oliguria after the postoperative period, connected to the irreversibility of renal failure (imaging tests suggested loss of the corticomedullary relationship).

Biopsy of the mass guided by USG was performed, whose anatomopathological evidence proved hemangioendothelioma.

The patient underwent pulmonary tomographic staging. Solid, non-calcified pulmonary nodules smaller than $0.5 \mathrm{~cm}$ were found, which were not pathognomonic of malignancy after complementary evaluation of the thoracic surgery, allowing surgical resection of the tumor in April 2018.

The access of the surgery was through a median infraumbilical laparotomy incision. Dissection of the tumor from the prostatic and bladder planes was made, with no signs of peritoneal implants or local invasion. The tumor was removed without violation, and maintained those characteristics of a thin capsule and liquefied content, and we performed a pelvic iliac obturator lymphadenectomy for local staging.

Anatomopathological revealed high-grade hemangioendothelioma. On macroscopy, a $9 \times 6 \times 6 \mathrm{~cm}$ ovoid tumor weighing $147 \mathrm{~g}$ had a smooth, shiny surface and permeable fat tissue (Figure 2). Inside, the appearance was solid-cystic and filled with hemorrhagic material. On microscopy, the sections showed a neoplasm characterized by extraordinary cell proliferation involving small vascular gaps of the endothelium with a marked degree of polymorphism and nuclear atypia, absence of tumor necrosis, and

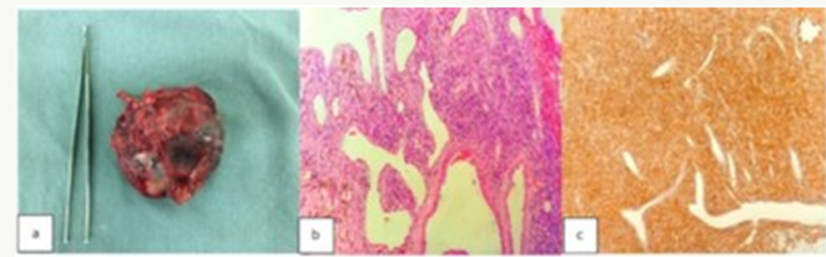

Figure 2. A. Hemangioendotheliomas macroscopy; B. Proliferation of spindle cells areas with small capillary, associated with large lymphatic channels (Hemangioendothelioma); C. Immunostains positive for CD34 (hemangioendothelioma). absence of metastasis in resected lymph nodes. In the immunohistochemical study, CD34 was positive, proving the endothelial nature of the neoplasm (Figure 3).



Figure 3. Axial and coronal section, respectively, of contrasted computed tomography of the abdomen and pelvis showing a $15 \times 11 \mathrm{~cm}$ retroperitoneal tumor, well-defined and with a solid component.

The patient was discharged after removal of the drain on the $5^{\text {th }}$ day but remaining on scheduled outpatient dialysis ( 3 times a week) due to irreversibility of chronic kidney disease and low urine output. During the outpatient segment, he presented urinary retention after attempting to remove the bladder catheter. An underactive bladder was diagnosed and possibly assigned to a lesion of the sacral plexus due to a tumorous mass effect after carrying out a urodynamic study.

During oncological monitoring, in April 2019, abdomen and pelvis CT showed a $15 \times 11 \mathrm{~cm}$ retroperitoneal tumor (Figure 3 ) with no signs of systemic metastasis. The patient was subjected to a new surgical procedure, in June 2019, for the resection of the tumor. He underwent xyphopubic laparotomy, removal of 2,141g tumor without capsule violation, no signs of adhesions, and no peritoneum implants.

Anatomopathological after theimmunohistochemical examination wascompatiblewith myxoid liposarcoma (Figure 4), a surprising finding due to the fact of a previous different neoplastic histological type. We reviewed the slides of the three histological pieces (biopsy and anatomopathological of both tumors) in the department of pathology of Santa Casa de Sao Paulo, confirming the results.

The patient's outcome was a cerebral ischemic stroke in the immediate postoperative period, ending in death one month after the surgical procedure, due



Figure 4. A. Surgical specimen after resection (myxoid liposarcoma); B. Myxoid liposarcoma: there is a mixture of lipoblasts cells and a prominent myxoid stroma; C. Immunostains positive for S100 protein. 
to clinical complications secondary to neurological damage and previously impaired renal function.

\section{DISCUSSION}

Hemangioendothelioma is a rare type of vascular neoplasia that can present as a benign or malignant form. There are six major histopathological variants described in the literature (Table 1). ${ }^{[4]}$

Table 1. Main histopathological variants of hemangioendotheliomas.

Intralymphatic papillary hemangioendothelioma
Retiform hemangioendothelioma
Kaposiform hemangioendothelioma
Epitheliod hemangioendothelioma
Pseudomyogenic
Hemangioendothelioma compound
Hemangioendothelioma

Themostcommonaffectionofhemangioendothelioma is in the liver (21\%), liver and lung (18\%), lung alone (12\%), and bone alone (14\%). However, hemangioendothelioma is a very heterogeneous tumor, and it is involved in many other sites..$^{[1,4]}$ The reported case shows a rare form of presentation, which is hemangioendothelioma in the pelvic region.

Histologically, hemangioendothelioma presents epithelioid cells with intracytoplasmic vacuoles in a hyalinized or mucinous stroma. Spindle cells or giant cells similar to osteoclasts can occasionally be seen. [1] Immunohistochemistry demonstrates endothelial differentiation (positivity of factor VIII in $99 \%$ of cases, CD34 in 94\%, CD31 in 86\%, cytokeratin rarely, and richness of collagen and type IV laminin). ${ }^{[5,6]}$

There are possible etiologies and risk factors for the disease, such as vinyl chloride, polyurethane/silica exposure, oral contraceptives, primary biliary cirrhosis, viral hepatitis, use of asbestos, and alcohol, but no solid evidence. ${ }^{[5]}$ Most studies indicate that there is a higher incidence in females (male/female: 1:4), except pleural hemangioendothelioma, which is more frequent in males. It usually affects middle-aged patients, although there are reported cases in children and the elderly. The usual age of diagnosis varies from 20 to 60 years. ${ }^{[1]}$ This case reports an unusual age range and atypical location in a male patient.

Early diagnosis does not usually happen due to the rarity of the tumor, so the index of suspicion is low. [3] They tend to be asymptomatic or with non-specific symptoms. ${ }^{[5]}$ Also, it grows insidiously and silently, causing no symptoms in the early stages. ${ }^{[3]}$

The reported patient came to present symptoms due to the size of the tumor that compressed adjacent structures, causing extensive compression of the urinary tract, progressing to a stage of renal failure. This fact demonstrates an already advanced phase of the tumor, considering that its growth is slow and does not usually present symptoms in the initial stages.
Regardingimagingdiagnosis, hemangioendothelioma is shown as hypoechogenic lesions on ultrasound and hypodense on non-contrast tomography. At resonance, it presents a low signal at T1, a high/ intermediate signal at $\mathrm{T} 2$, and it is possible to identify a targeting pattern with a central high-signal area. The central portion with low signal may be due to hemorrhage, calcification, and coagulative necrosis. In both tomography and magnetic resonance imaging, the nodules have a hypovascular and peripheral enhancement pattern. ${ }^{[7]}$ The definitive diagnosis is only possible through histopathological examination and confirmed by an immunohistochemical study of fragments obtained from biopsy or after surgical resection of the lesion. ${ }^{[3]}$

Surgical resection of the tumor is the treatment of choice in non-metastatic disease, especially in minor or welllocated lesions. Successful curative resection achieves good results. The role of adjuvant chemotherapy and or radiotherapy (RT) is not yet well defined. RT is chosen after surgical resection for localized hemangioendotheliomas to control residual disease (due to its recurrence); however, the response of these tumors to radiotherapy is frustrating. ${ }^{[1,3]}$ Some studies indicate the use of chemotherapy with thalidomide in the treatment of hepatic hemangioendothelioma, and it works as adjuvant therapy after surgical resection, but further studies are needed to prove the real benefit of this drug as an adjuvant treatment in case of hemangioendotheliomas from other sites. ${ }^{[8]}$

Another chemotherapeutic agent that has been showing benefits is sirolimus. A retrospective analysis from the Italian Rare Cancer showed that there was a partial response in 17 evaluated patients, 12 of which had stable disease. The rate of clinical benefit (objective response plus stable condition for at least six months) was 56\%, which lasted for more than 24 months in four. ${ }^{[9]}$

Treatment of metastatic disease included cytotoxic chemotherapy, immunotherapy, and targeted therapies. An open, multicenter, phase 2 study using bevacizumab for the treatment of angiosarcoma and hemangioendothelioma showed that bevacizumab is effective and well-tolerated alone and should be considered for combination with other chemotherapeutic agents in the future, as $17 \%$ of patients had a partial response and $50 \%$ showed stable disease with an average progression of 26 weeks. ${ }^{[6]}$

A study on renal hemangioendothelioma suggested that ultrasound follow-ups can take place after surgical resection of these tumors, although there is no consensus on which exam is the best followup. ${ }^{[10]}$ The prognosis is uncertain. Some cases show indolent behavior, while others may present a more aggressive character, leading to death within a short period after diagnosis. ${ }^{[4]}$ In cases of low-grade hemangioendothelioma, survival can reach a few decades after diagnosis (a case report of a 24-year survival). However, the survival rate is not longer than a few weeks after the diagnosis in those with a highdegree malignancy (as seen in angiosarcoma). ${ }^{[11]}$ 
The reported patient had a disease-free survival of one year, presenting a recurrence with a histologically different tumor after this period, a case not yet reported in the current medical literature, as the authors know so far. To better explain this fact, the genetic part of the neoplasm was taken into account, resulting in the hemangioendothelioma characterized by recurrent translocations involving the chromosomal regions 1 p36.3 and 3q25, whereas myxoid liposarcoma presents $\mathrm{t}(12 ; 16)(\mathrm{q} 13 ; \mathrm{p} 11)$ translocation, presenting as tumors with different genetic alterations. There are no studies that indicate the appearance of these two tumors synchronously or even that they show recurrence of hemangioendothelioma as another form of tumor such as myxoid liposarcoma. ${ }^{[2]}$ The analysis of gene expression is a crucial tool to guide the diagnosis, treatment, and assessment regarding the risk of recurrence, given the rarity of the disease. ${ }^{[6]}$

Our case report highlights an unusual clinical presentation, etiopathogenesis, diagnosis, and treatment of pelvic hemangioendothelioma. Due to the rarity of the tumor, further studies are needed to understand its etiopathogenesis, as well as better defining the most effective therapeutic approaches.

\section{REFERENCES}

1. Sardaro A, Bardoscia L, Petruzzelli MF, Portaluri M. Epithelioid hemangioendothelioma: an overview and update on a rare vascular tumor. Oncol Rev. 2014 Oct;8(2):259.

2. DeLaney TF, Kirsch DG. Pathogenetic factors in soft tissue and bone sarcomas. UpToDate [Internet]. 2019; [access in 2019 Dec 15]. Available from: https://www.uptodate.com/ contents/pathogenetic-factors-in-soft-tissueand-bone-sarcomas\#: : :text=There $\% 20$ is $\% 20$ no\%20clearly\%20defined,\%2C\%20chronic\%20 irritation\%2C\%20and\%20lymphedema
3. Lopes T, Clemente S, Feliciano A, Lourenço I, Costa A. Pulmonary epithelioid hemangioendothelioma - rarity, diagnostic and tratment difficulties. Rev Port Pulmonol. 2009 Nov/Dec;15(6):1167-74.

4. Dutta R, Pal H, Garg G, Mohanty S. An aggressive large epithelioid hemangioendothelioma of the anterior mediastinum in a young woman. Korean J Thoracic Cardiovasc Surg. 2018 Dec;51(6):419-22.

5. Llueca A, Piquer D, Maazouzi Y, Medina C, Delgado K, Serra A, et al. Hepatic epithelioid hemangioendothelioma: a great mimicker. Int J Surg Case Rep. 2018;53:25-8.

6. Patel E, Kancharla P, Surapaneni BK, Hennrick $\mathrm{K}$, Goldfinger M, Kalavar M. A case of vanishing metastatic mass: right atrial mass in the setting of primary epithelioid hemangioendothelioma of the spine. Case Rep Oncol. 2018;11:534-50.

7. Torres LR, Timbó LS, Ribeiro CMF, Galvão Filho MM, Verrastro CGY, D'Ippolito G. Multifocal and metastatic hepatic hemangioendothelioma: case report and literature review. Radiol Bras. 2014 May;47(3):194-6.

8. Wang J, Wu ZR, Su F, Ma WJ, Hu HJ, Li FY. Resectable single hepatic epithelioid hemangioendothelioma in the left lobe of the liver: a case report. Open Med (Wars). 2018;13:456-9.

9. George S. Systemic treatment of metastatic soft tissue sarcoma. UpToDate [Internet]. 2019; [access in 2019 Dec 23]. Available from: https:// www.uptodate.com/contents/systemic-treatment-of-metastatic-soft-tissue-sarcoma

10. Liu Y, Liu A, Wu J, Liu T. Epithelioid hemangioendothelioma arising from the kidney. Medicine (Baltimore). 2019 Aug;98(34):e16537.

11. Heera R, Cherian LM, Lav R, Ravikumar V. Hemangioendothelioma of palate: a case report with review of literature. J Oral Walks Maxillofac Pathol. 2017 Sep/Dec;21(3):415-20. 